\title{
Comment on "Quasiperiodic spin-orbit motion and spin tunes in storage rings"
}

\author{
S. Y. Lee ${ }^{1}$ and S. R. Mane ${ }^{2}$ \\ ${ }^{1}$ Department of Physics, Indiana University, Bloomington, Indiana 47405, USA \\ ${ }^{2}$ Convergent Computing Inc., P.O. Box 561, Shoreham, New York 11786, USA
}

(Received 28 December 2004; published 2 August 2005)

\begin{abstract}
Contrary to the claim of the recent publication by Barber, Ellison, and Heinemann [Phys. Rev. ST Accel. Beams 7, 124002 (2004).], we explain in this Comment that (1) the snake resonances are spin depolarizing resonances just like other spin depolarizing resonances and (2) the perturbed spin tune is useful to understand depolarization phenomena.
\end{abstract}

DOI: $10.1103 /$ PhysRevSTAB.8.089001

Recently, we received a publication of a paper by Barber, Ellison, and Heinemann (BEH) on quasiperiodic spin-orbit motion and spin tunes in storage rings [1]. While we welcome their ideas, we note that BEH make criticisms of calculations that both of us have published (sometimes in collaboration with coauthors), e.g., that the use of the "perturbed spin tune" (to be defined below) may cause confusion in the literature. We are not aware of any confusion caused by our papers. In particular, nobody has published a wrong calculation because of the concepts and definitions in our papers. We also point out, contrary to the claim of BEH, that the so-called "snake resonances" (depolarizing resonances in rings equipped with Siberian snakes) are just like other spin depolarizing resonances, and can be understood in terms of conventional spin-orbit coupling theory.

In fact, we can point to useful achievements which have resulted from our work. Lee and Tepikian [2] predicted the existence of the snake resonances (with later work by Lee on even-order snake resonances [3]; see also [4]). Snake resonances were observed experimentally at the Indiana University Cyclotron Facility (IUCF) Cooler [5] and later at the Relativistic Heavy Ion Collider (RHIC) [6]. The observations are in conformity with the theoretical calculations. The snake resonances were independently rederived by Mane [7], who gave a nonperturbative analytical solution in terms of so-called "sine-Bessel" functions. The sine-Bessel functions are now used to benchmark the spintracking codes at BNL [8].

We summarize the principal details of our work. The spin precession for the rest-frame spin vector $\vec{S}$ is governed by the Thomas Bargmann-Michel-Telegdi equation [9]

$$
\frac{d \vec{S}}{d \theta}=\vec{\Omega} \times \vec{S},
$$

where $\theta$ is the ring azimh autnd the spin precession vector $\vec{\Omega}$ depends on the electromagnetic fields of the accelerator and the particle's anomalous magnetic $g$ factor $G=(g-$ $2) / 2$, mass, charge, and velocity. It is well known that a beam can suffer depolarization during acceleration and storage due to so-called "depolarizing spin resonances."
PACS numbers: 29.27.Hj, 29.20.Dh, 02.60.Lj, 02.30.Nw

In general, such resonances occur when the spin precession is coherently perturbed by the imperfection and (off-axis) intrinsic fields in the accelerator. Derbenev and Kondratenko proposed special spin rotators, called "Siberian snakes," to alleviate this agonizing situation [10]. With snakes, the (closed orbit) spin tune can be made exactly $1 / 2$ at all beam energies. Siberian snakes therefore offer the possibility that a polarized beam can, in principle, be accelerated to arbitrarily high energies without crossing any depolarizing resonances.

However, depending on the magnitude of the driving terms in the spin precession vector, the spins may still be depolarized in the ring. We analyze a simple model for the spin motion in an accelerator with two snakes and one isolated depolarizing spin resonance, where the spin precession vector is

$$
\begin{aligned}
\vec{\Omega}= & G \gamma \hat{e}_{3}+\epsilon\left(\hat{e}_{1} \cos \phi+\hat{e}_{2} \sin \phi\right)+\pi \delta_{p}(\theta) \hat{e}_{1} \\
& +\pi \delta_{p}(\theta-\pi) \hat{e}_{2} .
\end{aligned}
$$

Here $\left(\hat{e}_{1}, \hat{e}_{2}, \hat{e}_{3}\right)$ are unit vectors in the radial, longitudinal, and vertical directions, respectively, $\epsilon$ is the magnitude of the resonance driving term, $\phi$ is related to the synchrobetatron phase advance, e.g., $d \phi / d \theta=K$ with $K=$ half integer, and the periodic $\delta$ function is

$$
\delta_{p}\left(\theta-\theta_{0}\right)=\sum_{j=-\infty}^{\infty} \delta\left(\theta-\theta_{0}-2 \pi j\right) .
$$

The above model (with small changes of notation) was the basis for the analysis in Refs. [2,3]. Since the spin tune is $\nu_{s}=1 / 2$, the resonance condition of $\nu_{s}=K$ never occurs. Unfortunately, when $|\epsilon|$ is large, the beam can still be depolarized when the "snake resonance" condition

$$
\nu_{s}-\ell K=\text { integer } \quad(\ell=\text { integer })
$$

is met. The above snake resonance conditions were derived based on perturbation theory of a spin-tracking hierarchy of equations, and confirmed by numerical spin-tracking calculations. Subsequently, Mane [7] employed Hamiltonian dynamics to solve the model in Eq. (2) to derive a nonperturbative solution for the $\hat{n}$ axis, and con- 
firmed the findings in Ref. [2]. As stated above, experimental observations of snake resonances have been made at the IUCF Cooler Ring [5] and RHIC [6].

We now arrive at what seems to be the heart of the criticisms in BEH. Although the spin tune (as defined in the sense of Hamiltonian dynamics) is $1 / 2$ with snakes, the spin precession is perturbed by the existence of the spin depolarizing resonances. One of us (S. Y. L.) introduced the concept of the "perturbed spin tune" from the trace of the spin one-turn map to understand the snake resonance phenomena $[3,4]$. The key to BEH seems to be restrictions they place on the usage of the words "spin tune." It appears that $\mathrm{BEH}$ is intended to call foul to the definition of the perturbed spin tune as a "fake spin tune" [11], and hides the snake resonances via statements such as "static phenomenon characterized by an invariant spin field which is irreducibly discontinuous in $\phi_{2}$ " [11].

Our Comment is intended to clarify the point that a snake resonance is a depolarizing spin resonance just like any other, e.g., the imperfection and intrinsic resonances which have long been known in the literature. It is recognized that the "perturbed spin tune" is not a secular spin tune in the sense of Hamiltonian dynamics, and was never claimed to be so. Indeed, Mane [7] performed his calculations entirely within the framework of Hamiltonian dynamics, and derived a solution for the secular spin tune, which he showed is $1 / 2$ on all of the "well-tuned tori" as defined by BEH. (Mane treated both the one-snake and two-snake models. He also solved the single snake model using Yokoya's SODOM2 formalism [12].) Yet, the perturbed spin tune, representing the phase advance of the spin vector in each succeeding revolution, is useful to understand some depolarization phenomena to be addressed below. The analytic solution of the $\hat{n}$ axis for the two-snake model in Eq. (2) was obtained as a Fourier series

$$
n_{3}=A_{0}+2 \sum_{m=\text { even }} A_{m} \cos m \phi_{*} .
$$

Here the $A_{m}$ are sine-Bessel functions, and $\phi_{*}$ is the value of $\phi \sim K \theta$ at an observation point (e.g., just before the first snake). The snake resonance spectrum is given by the locations of the singularities in the expression for $n_{3}$, i.e., the sine-Bessel functions, and agrees with that published in [2]. A quantity of interest is $\left\langle n_{3}\right\rangle$, with a uniform average over the phase $\phi_{*}$ (i.e., an ensemble average for particles with all betatron phases). From Eq. (5), we find

$$
\left\langle n_{3}\right\rangle=A_{0}
$$

Ptitsin and Shatunov [13] has also solved this model, to leading order in perturbation theory (using standard spinorbit theory). Their expression for $\left\langle n_{3}\right\rangle$ is equivalent to Eq. (6), within the approximations they make.

We now briefly discuss the perturbation theory in [2]. The $n$-turn spin map $T\left(\theta_{n}\right)$ is calculated iteratively by using the spin-tracking equation

$$
T\left(\theta_{n+1}\right)=\tau\left(\theta_{n+1}, \theta_{n}\right) T\left(\theta_{n}\right),
$$

where $\theta_{n+1}=\theta_{n}+2 \pi$. Here $\tau\left(\theta_{n+1}, \theta_{n}\right)$ is the one-turnmap (see [2] for details). The equations can be solved using a power series expansion in the strength parameter $b^{2}$, where $b=(\epsilon / \lambda) \sin (\pi \lambda / 2)$ and $\lambda=\sqrt{(K-G \gamma)^{2}+\epsilon^{2}}$. Then

$$
\begin{aligned}
& T_{11}=T_{11}^{(0)}+T_{11}^{(1)}+T_{11}^{(2)}+\cdots, \\
& T_{12}=T_{12}^{(1)}+T_{12}^{(2)}+T_{12}^{(3)}+\cdots,
\end{aligned}
$$

where $T_{11}^{(i)}=O\left(b^{2 i}\right)$ and $T_{12}^{(i)}=O\left(a b^{2 i-1}\right)$, where $a=$ $\sqrt{1-b^{2}}$. The polarization is given by $\left\langle S_{z}\right\rangle=1-$ $2\left|T_{12}\right|^{2}$. The off-diagonal spin transfer matrix element $T_{12}$ for the $\ell$ th order snake resonances is a result from the coherent perturbing kicks, that can be summed to

$$
T_{12}^{(\ell)}\left(\theta_{n}\right) \sim b^{2 \ell-1} \frac{\sin \left[n \pi\left(\ell K \pm \nu_{s}\right)\right]}{\sin \left[\pi\left(\ell K \pm \nu_{s}\right)\right]} .
$$

On a snake resonance, the spin vector is perturbed coherently, and the perturbative solution diverges, a typical characteristic of any physical system encountering a resonance.

The spin-tracking hierarchy was not used in Refs. [2-4] to solve for the $\hat{n}$ axis (but in principle it can be adapted to do so). One defines the operational "perturbed spin tune" $Q_{s}$ as

$$
\cos \left[\pi Q_{s}(n)\right]=\frac{1}{2} \operatorname{Trace}\left[\tau\left(\theta_{n+1}, \theta_{n}\right)\right]=b^{2} \sin (2 \phi),
$$

where $\phi$ depends on a combination of the resonance tune and orbital angle $K \theta$. For an intrinsic spin resonance, $K$ is not an integer, and thus the perturbed spin tune oscillates around the spin tune of $1 / 2$ with an envelope given by

$$
Q_{s, \max / \min }=\frac{1}{2} \pm \frac{1}{\pi} \arcsin b^{2} .
$$

Since the betatron motion of a particle has a different betatron phase in each succeeding turn, the spin phase advance in each revolution, perturbed by existence of spin resonances, also changes accordingly. If one waits long enough to trace all betatron orbits, the betatron orbit can return its original position. This explains the time average and ensemble average produce the same spin tune of $1 / 2$.

We can apply the perturbed spin tune to the single resonance model with two snakes [see Eq. (2)]. With snakes, the spin tune becomes $1 / 2$ and the effective spin perturbing kick in each snake section becomes $b$ at the spin kick tune of $K$. Because of the perturbed spin kick, the phase advance of the spin vector is modified to $Q_{s}$, which is unfortunately complicated by its turn-by-turn variation. Taking the $Q_{s, \max } / \min$ as the dominant effect on spin motion, we derive 


$$
\left\langle S_{3}\right\rangle=\frac{\delta}{\sqrt{\delta^{2}+\epsilon_{\mathrm{eff}}^{2}}},
$$

where $\epsilon_{\text {eff }}=2 b / \pi$ and $\delta=-\left(K-Q_{s, \max / \min }\right)$ (the extrema of the perturbed spin tune). Figure 1 compares the approximate expression of Eq. (13) (dashed line) against $\left\langle n_{3}\right\rangle$ of Eq. (6) (solid line), where $\left\langle n_{3}\right\rangle=A_{0}$ and $\left\langle S_{3}\right\rangle$ are plotted against $K$ for $0 \leq K \leq 0.5$, for three values $\epsilon=$ $0.2,0.4$, and 0.6. When the resonance tune falls within the range of the perturbed spin tune, the spin precession becomes complicated, one cannot derive a simple approximate formula. Figure 1 shows that the use of the perturbed spin tune can give a useful approximate expression for the polarization. More graphs can be found in [14], where, for example, it is shown that a numerical calculation of the polarization using stroboscopic averaging [15] yields identical results to Eq. (6).

Now we consider the spin motion of a particle in an accelerator with two snakes and an imperfection resonance $\left(\epsilon_{\mathrm{imp}}=0.36\right.$ at $\left.K_{\mathrm{imp}}=485\right)$ and a very weak intrinsic spin resonance $\left(\epsilon_{\text {int }}=0.0033, K_{\text {int }}=485+K\right)$. The particle energy is accelerated from far below to far above the resonance region $G \gamma \simeq 485$. Because the intrinsic resonance strength is very small, the perturbed spin tune $Q_{s}$ is essentially produced by the imperfection resonance. The

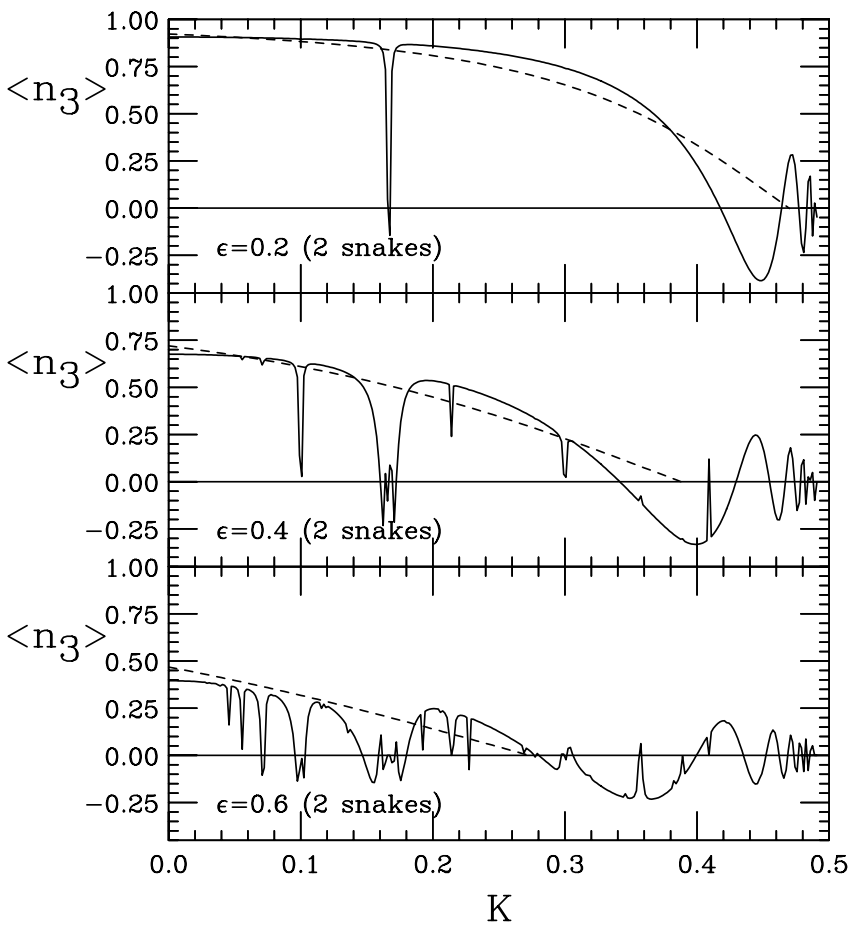

FIG. 1. The expression $\left\langle n_{3}\right\rangle=A_{0}$ (solid curve), is compared with an approximate expression for $\left\langle S_{3}\right\rangle$ (dashed curve) calculated using the perturbed spin tune, as a function of the resonance tune $K(K=0$ to 0.5$)$ for a single resonance model with two snakes. Three plots are shown, for resonance driving terms with amplitudes $\epsilon=0.2$ (top plot), 0.4 (center), and 0.6 (bottom). lower plot of Fig. 2 shows the perturbed spin tune $Q_{s}$, and the top plot shows the polarization, $\left\langle S_{z}\right\rangle$, after passing through these two spin resonances for three intrinsic tune values $K=0.59,0.60$, and 0.61 . When the intrinsic resonance tune $(K=0.61)$ does not overlap with the perturbed spin tune, there is no depolarization. However, when the intrinsic spin resonance tune overlaps with the perturbed spin tune, the spin is depolarized by the spin resonance. The concept of the perturbed spin tune is well-defined for an accelerating beam.

Another example (which we acknowledge was known by others before our works, and does not involve snakes, and did not employ the name "perturbed spin tune") is the perturbation of the spin tune in a planar ring caused by synchrotron oscillations $\nu_{s}=G \gamma_{0}\left(1+\Delta E / E_{0}\right)$, resulting in multiple crossings of a resonance line during acceleration. This phenomenon has been experimentally observed at several synchrotrons.

In conclusion, we have listed several formalisms, by different authors, which all treat the polarization in rings equipped with snakes within the framework of existing spin-orbit coupling theory. The formalisms are all in agreement with each other, within the framework of the various approximations made. We also presented a brief description on the use of the perturbed spin tune. The perturbed spin tune, defined as the number of spin precession turns per orbital revolution, is naturally one of relevant parame-

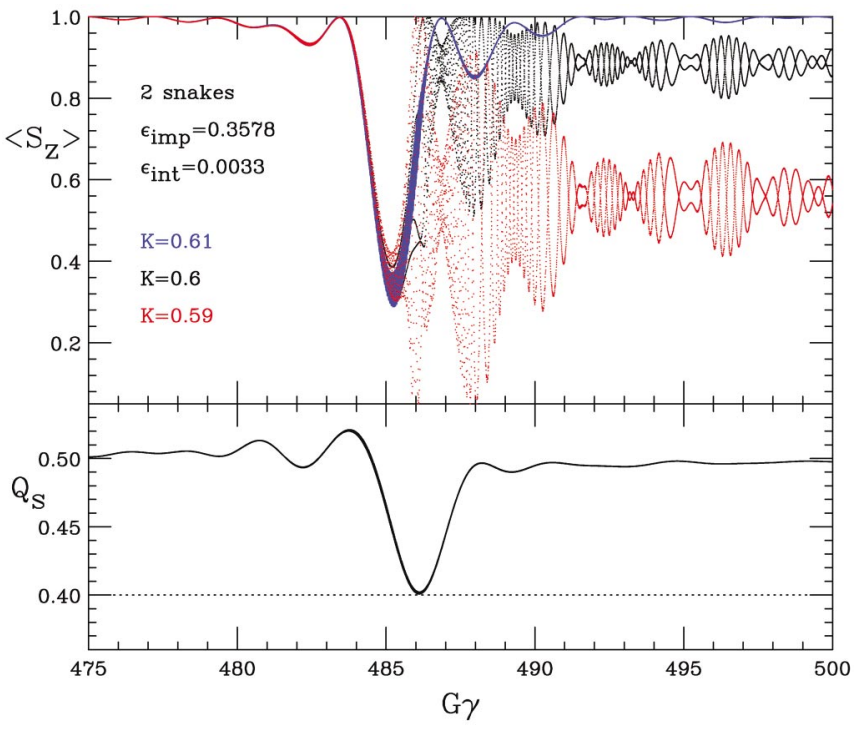

FIG. 2. (Color) The effect of depolarization due to perturbed spin tune shift on the spin. The model contains an imperfection and a weak intrinsic spin resonances in an accelerator with two snakes. The top plot shows the value of $\left\langle S_{z}\right\rangle$ after acceleration across the resonance region. The bottom plot shows the perturbed spin tune $Q_{s}$. Plots for three intrinsic tune values $K=$ $0.59,0.6$, and 0.61 are shown. Note that the imperfection spin resonance produces a spin tune shift, and the depolarization is clearly observed when the tune of the intrinsic spin resonance overlaps with the perturbed spin tune. 
ters that determines the spin depolarization in passing through spin resonances. We have offered evidence that snake resonances are none other than standard spin depolarizing resonances. We have no objection to the definitions of BEH. We see no evidence that their definitions lead to different answers for the snake resonances.

\section{ACKNOWLEDGMENTS}

This work is supported in part by grants from the U.S. Department of Energy: US DOE DE-FG0292ER40747, and NSF PHY-0244793.

[1] D. P. Barber, J. A. Ellison, and K. Heinemann, Phys. Rev. ST Accel. Beams 7, 124002 (2004).

[2] S. Y. Lee and S. Tepikian, Phys. Rev. Lett. 56, 1635 (1986).

[3] S. Y. Lee, Phys. Rev. E 47, 3631 (1993).

[4] S. Y. Lee, Spin Dynamics and Snakes in Synchrotrons (World Scientific, Singapore, 1997).

[5] R. A. Phelps et al., Phys. Rev. Lett. 78, 2772 (1997).

[6] V. H. Ranjbar et al., Phys. Rev. Lett. 91, 034801 (2003). More recent measurements are given in V. Ptitsin et al.,
Proceedings of SPIN 2004 (World Scientific, Singapore, to be published).

[7] S. R. Mane, Nucl. Instrum. Methods Phys. Res., Sect. A 498, 1 (2003).

[8] T. Roser and S. Tepikian (private communication).

[9] L. H. Thomas, Philos. Mag. 3, 1 (1927); in AIP Conf. Proc. No. 95 (AIP, N.Y., 1983), p. 4; V. Bargmann, L. Michel, and V. L. Telegdi, Phys. Rev. Lett. 2, 435 (1959).

[10] Ya. S. Derbenev and A. M. Kondratenko, Sov. Phys. Dokl. 20, 562 (1976).

[11] D. P. Barber, R. Jaganathan, and M. Vogt, in SPIN 2002: 15th International Spin Physics Symposium and Workshop on Polarized Electron Sources and Polarimeters, AIP Conf. Proc. No. 675 (AIP, New York, 2003), p. 741.

[12] K. Yokoya, DESY Report No. 99-006, 1999; see also physics/9902068.

[13] V. Ptitsin and Yu. M. Shatunov, in Proceedings of the 12th International Symposium on High-Energy Spin Physics, edited by C.W. de Jager et al. (World Scientific, Singapore, 1997), p. 516.

[14] S. R. Mane, Nucl. Instrum. Methods Phys. Res., Sect. A 528, 677 (2004).

[15] K. Heinemann and G. H. Hoffstätter, Phys. Rev. E 54, 4240 (1996). 